\title{
STRONGLY ASYMMETRIC BEAMS AT THE UNIVERSITY OF MARYLAND ELECTRON RING (UMER)*
}

\author{
S. Bernal ${ }^{\dagger}$, R.A. Kishek, B. Quinn, M. Reiser, M. Walter, and P.G. O'Shea, \\ IREAP, University of Maryland, College Park, MD USA
}

\begin{abstract}
The standard operation of the University of Maryland electron ring (UMER) employs symmetric strong focusing with magnetic quadrupoles, i.e., a FODO scheme whereby the zero-current betatron phase advances per period in the two transverse planes are equal or nearly so. Asymmetric focusing, on the other hand, employs quadrupoles with different strengths in a FODO cell. Typically, a small focusing asymmetry is implemented in most accelerators to set the operating point (horizontal and vertical zero-current tunes) in order to avoid resonances and/or compensate for edge focusing of bending magnets. Extreme asymmetry, however, is rarely, if at all, used. We review the motivation and theory of beam transport with general focusing asymmetry. We also present preliminary results of an experiment with highly asymmetric focusing of a space-charge dominated electron beam in UMER.
\end{abstract}

\section{INTRODUCTION}

The study of beam instabilities plays a crucial role in the design of accelerators, as beam instability is one of the main factors leading to emittance growth and halo formation. The field has become the subject of intensive study since at least 1970, when Gluckstern analyzed the stability properties of isotropic, continuous beams in the framework of the Kapchinskij-Vladimirskij (K-V) distribution in a uniform-focusing lattice [1]. An extension of Gluckstern's work to periodic solenoid and quadrupole focusing by Hofmann et al [2] revealed additional instabilities. More recent work by Hofmann [3] has considered the stability of continuous anisotropic $\mathrm{K}-\mathrm{V}$ beams in the context of the uniform-focusing approximation. Highly anisotropic beams in linacs are predicted to be stable even in the presence of moderate space charge. Synchrotrons and storage rings, on the norther hand, operate at much lower intensity than linacs [4]. The University of Maryland Electron Ring (UMER), by contrast, is designed to operate like a "recirculating linac", with tune depressions far exceeding those in any ring or even the highest-intensity linacs.

The motivation and commissioning plan of UMER are discussed in these proceedings [5]. In this paper, we sketch the design of experiments with anisotropic beams in UMER over a broad range of intensities. We also present preliminary results of an experiment with an anisotropic, space-charge dominated beam. The anisotropy arises from

\footnotetext{
* Work supported by the U.S. Department of Energy

† sabern@umd.edu
}

different average beam sizes obtained with an asymmetric FODO scheme, i.e., whereby the zero-current phase advances per period, $\sigma_{0 x}, \sigma_{0 y}$, as set up by external focusing, are significantly different. It is assumed that the emittances in the transverse directions are equal: $\epsilon_{x}=\epsilon_{y}$. This assumption is supported, within experimental error, by direct emittance measurements.

In the last section, we compare general features of beam transport experiments employing symmetric and asymmetric FODO focusing, over a transport distance of 20 FODO full periods, or $7.0 \mathrm{~m}$, approximately (almost 2/3-turn). While the experiment described below does not provide definite answers about the sustainability of similar asymmetric transport in a ring lattice, it hints at general features of future similar experiments. A detailed stability analysis of anisotropic beams in UMER is left for a future study.

\section{ASYMMETRIC FODO TRANSPORT}

The periodic lattice in UMER consists of 18 ring sections containing 2 FODO periods per section, i.e., four quadrupoles. Each section has also two bending dipoles and a center diagnostics chamber or an induction module (3 sections). Figure 1a illustrates the layout of a typical ring section. The diagnostics (Figure 1b) include a fast capacitive beam-position-monitor (BPM), and a $1.3 \mathrm{~cm}$ (dia.) fluorescent screen. The fluorescent screen diagnostic (FSD) is placed $2.7 \pm 0.1 \mathrm{~cm}$ from the center plane of the chamber; either the BPM or the FSD can be reproducibly inserted in the beam line by means of an actuator. The beam image on the FSD is reflected by a mirror at $45^{\circ}$, so the images can be observed and captured through a viewing window (Fig. 1). The FSD is a powerful tool for first-turn beam diagnostics, especially for those beams with relatively small envelope excursions ( $0.55 \mathrm{~mA}$ to $24 \mathrm{~mA}$ in Table I, below).

At a given energy, the electron beam currents in UMER can be varied in a number of ways. For convenience, we use collimating apertures in a rotatable plate at the output of the electron source. In this way, different beam intensities in the sense defined in [5] can be obtained. Although the beam's core density does not change through collimation, the relative weights of emittance and beam perveance do.

Asymmetric focusing can be simply implemented in the periodic lattice. We choose $\sigma_{0 x}=76^{0}, \sigma_{0 y}=60^{\circ}$., which corresponds [8] to focusing function peak values $k_{0 x}=221.5 m^{-2}, \quad k_{0 y}=198.8 m^{-2}$, or on-axis peak quadrupole gradients $7.50 \mathrm{G} / \mathrm{cm}$, and $6.74 \mathrm{G} / \mathrm{cm}$, respectively, for a $10 \mathrm{keV}$ beam. The matching solutions, however, depend sensitively on the beam current. (We only 
(a)

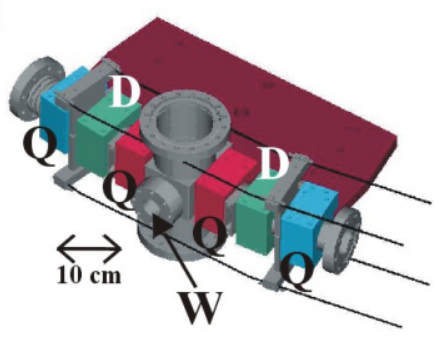

(b)

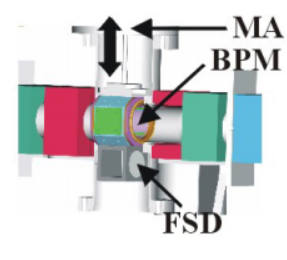

Figure 1: (a) Ring section in UMER showing four quadrupoles $(\mathrm{Q})$, two bending dipoles $(\mathrm{D})$, viewing window (W), and the center diagnostics chamber. (b) Cutout of center chamber. MA: mechanical actuator; BPM: beam position monitor; FSD: fluorescent screen diagnostic.

present one case in the next section). Following the method outlined in Ref. [8], we solve the K-V envelope equations in one FODO cell to extract the average beam sizes in the $x$ and $y$ directions, $a, b$, respectively. We also find the depressed phase advances within the "smooth-focusing" approximation applied independently to each transverse direction. It is also possible to consider a "smooth approximation" for the overall asymmetric case, as explained in Ref. [6].

The smooth-focusing approximation is useful for design calculations and is also the basis for Hofmann's stability analysis [3]. However, it is not clear which set of parameters is best for describing asymmetric beam transport. Hofmann introduces three parameters: the ratio of depressed betatron frequencies, the ratio of transverse emittances, and the beam ellipticity in real space. The product of tune ratio and emittance ratio defines the degree of "transverse anisotropy". Kishek [7], on the other hand, stipulates that a different set of parameters is more relevant: the ratio of zero-current phase advances, $\sigma_{0 y} / \sigma_{0 x}$, the ratio of total transverse kinetic to external field energies, and the ratio of initial kinetic energies in the two transverse directions. The latter parameter becomes identical to the ratio of depressed betatron frequencies if the initial transverse emittances are the same. At issue is which quantities are conserved, and which vary as a result of equipartition or energy/emittance exchange.

Without attempting to resolve the issues, we present in Table I a summary of beam parameters relevant to asymmetric FODO focusing in UMER for four cases of beam intensities. One case $(0.55 \mathrm{~mA})$ corresponds to emittancedominated beam transport, while the other three are spacecharge dominated.

Figure 2a shows the calculated envelope for the $7.2 \mathrm{~mA}$, $10 \mathrm{kev}$ case when focusing is symmetrical $\left(\sigma_{0 x}=\sigma_{0 y}=76^{0}\right)$. The asymmetric case $\left(\sigma_{0 x}=76^{0}, \sigma_{0 y}=60^{0}\right)$ is shown in Figure $2 \mathrm{~b}$. For the symmetrical case, the average beam radius is $a=b=3.0 \mathrm{~mm}$, approximately, while the asymmetrical case yields $a=2.9 \mathrm{~mm}, b=3.9 \mathrm{~mm}$. In case (a), a single tune depression close to 0.5 describes beam transport; in the second case, we have, from Table I, 0.52 and 0.37 for

Table 1: Parameters for asymmetric transport in $\mathrm{UMER}^{1}$

\begin{tabular}{|c|c|c||c|}
\hline Beam Current & Emitt. $^{2}$ & $b / a$ & $\sigma_{x} / \sigma_{0 x}, \sigma_{y} / \sigma_{0 y}$ \\
\hline $85 \mathrm{~mA}$ & $55 \mu \mathrm{m}$ & 1.6 & $0.22,0.12$ \\
24 & 30 & 1.5 & $0.36,0.23$ \\
7.2 & 16 & 1.4 & $0.52,0.37$ \\
0.55 & 6 & 1.2 & $0.86,0.80$ \\
\hline
\end{tabular}

${ }^{1} 10 \mathrm{keV}, \sigma_{0 x}=76^{0}, \sigma_{0 y}=60^{0}$.
2 4RMS, unnormalized.
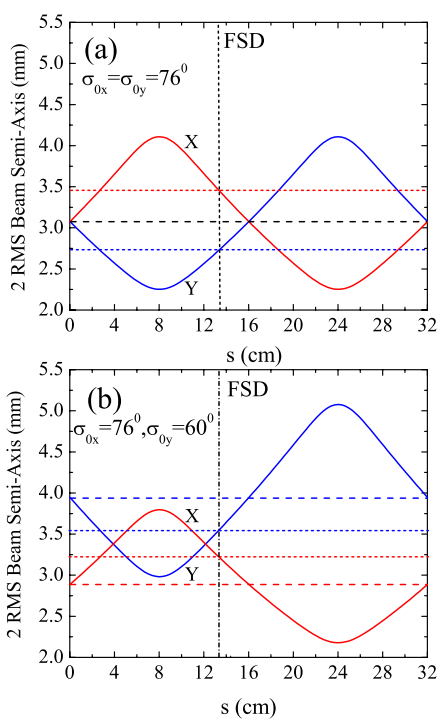

Figure 2: Calculated beam envelopes for a $7.2 \mathrm{~mA}, 10$ $\mathrm{keV}$ electron beam in (a) symmetric, and (b) asymmetric FODO cells. The vertical dashed lines indicate the location of the fluorescent screen diagnostic (FSD) in UMER. See also Fig. 1 and Table I.

the tune depressions associated with $x$ and $y$ motions, respectively. Thus, the beam is significantly more affected by space charge in the vertical $(y)$ plane.

\section{EXPERIMENT}

We implemented the asymmetric focusing described above, during experiments with DC injection. The matching/injection section used consists of a short solenoid, seven printed-circuit (PC) quadrupoles, one bending dipole and a number of steering and earth's-field compensation elements. The calculated envelope solution for matching in the asymmetric case is shown in Figure 3.

The results of beam size measurements for both symmetric and asymmetric focusing are shown in Figure 4. The measurements are based on captured video images of the beam in 10 ring chambers like the one shown in schematic form in Fig. 1. The experimental uncertainties arise mostly from image processing and calibration $(\mathrm{mm} / \mathrm{pixel})$. Despite the optimal video contrast used, background subtraction has to be approached on a picture-by-picture basis. The background can be as high as 8 in a gray scale 0-255 (8- 


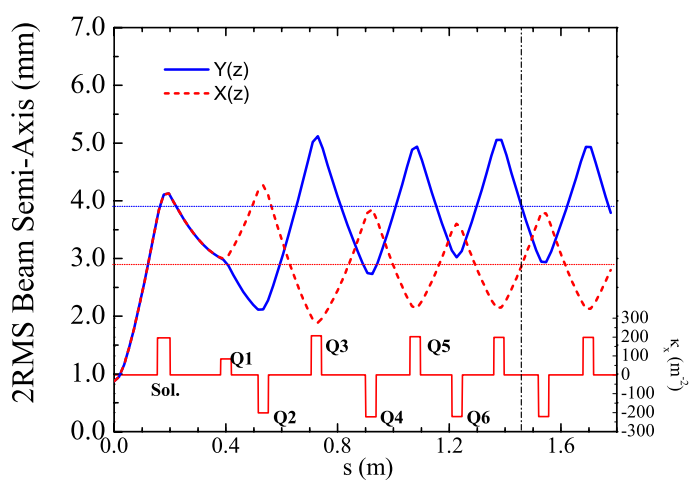

Figure 3: Calculated envelope and focusing function $\kappa_{x}\left(m^{-2}\right)$ for matching with asymmetric focusing $\left(\sigma_{0 x}=76^{0}, \sigma_{0 y}=60^{0}\right)$ of a $7.2 \mathrm{~mA}, 10 \mathrm{keV}, 16 \mu \mathrm{m}$ (edge emittance) electron beam.

bit pictures); in some cases, the calculated beam dimension depends sensitively on proper background subtraction. Halos pose a particular challenge in this regard, since their intensity is often not much stronger than the background.
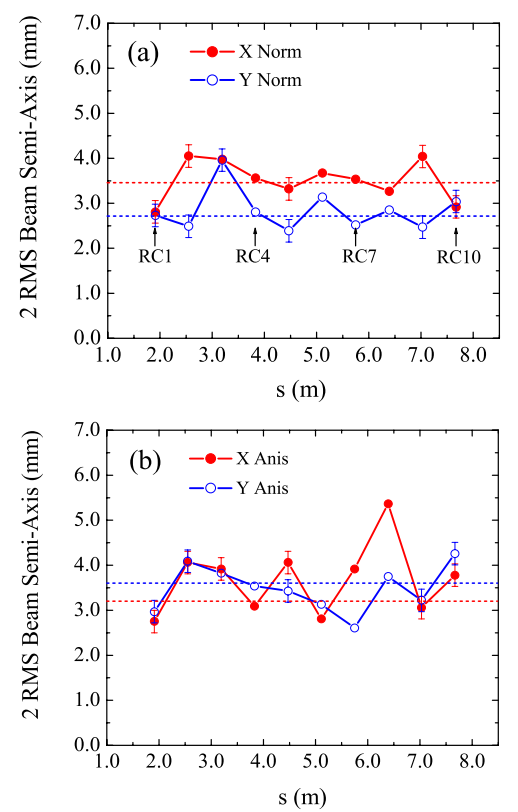

Figure 4: Measured electron beam $(7.2 \mathrm{~mA}, 10 \mathrm{keV})$ transverse dimensions in 10 ring chambers for: (a) symmetric focusing (labeled "Norm"), and (b) asymmetric focusing. The horizontal dotted lines correspond to the expected dimensions as read off the periodic envelopes in Fig. 2a-b at the plane of the diagnostics.

Mismatch oscillations are seen for both cases of focusing in Fig. 4. For symmetric focusing, Fig. 4a shows that the $y$-envelope oscillations become progressively smaller suggesting a "self-matching" beam. However, additional measurements are necessary, as the last data point seems to indicate a reversal in the trend. For asymmetric focusing (Fig $4 b$ ), the beam is initially (first three chambers) fairly round, but soon develops large excursions especially in the $x$-envelope. The latter also appears more erratic than the $y$-envelope in the symmetrical-focusing case, possibly indicating larger horizontal mismatch caused by edge focusing from the bending dipoles, which may be compounded by image forces from beam misalignment.

Another general feature in the beam evolution is the appearance of halos. Well defined halos are observed at some chambers when symmetrical focusing is employed, but become absent or completely different in character with asymmetrical focusing. Figure 5 illustrates this point at 4 ring chambers. The beam pictures shown use a logarithmic gray scale to enhance the low-intensity halos; the core appears "flat" as a consequence. Since the beam dynamics is fairly complex: mismatch, image forces, bending, quadrupole rotation errors, etc., we postpone further analysis to an expanded version of this article.

With the new pulsed injector in UMER, we have to reestablish a good first-turn base line for beam transport with symmetrical focusing before attempting new experiments with highly asymmetrical beams. To conclude, major questions remain about the sustainability of asymmetric transport in a ring lattice: how far can the beam go before significant energy exchange in the two transverse directions, i.e., equipartioning, and/or emittance exchange occur?

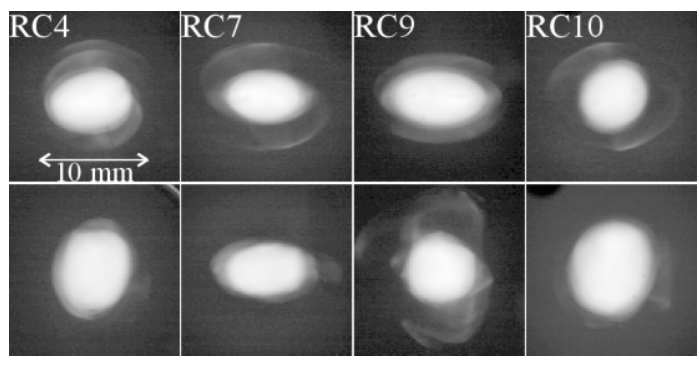

Figure 5: Logarithmic-grayscale rendering of electron beam $(7.2 \mathrm{~mA}, 10 \mathrm{keV})$ images at four chambers in UMER (see also Figs. 1 and 4). TOP ROW: symmetrical transverse focusing. BOTTOM ROW: highly asymmetrical transverse focusing.

\section{REFERENCES}

[1] R.L. Gluckstern, Proceedings of the linac Conference (Fermilab, Batavia, 1970), p. 811.

[2] I. Hofmann, L.J. Laslett, L. Smith, and I. Haber, Part. Accel. 13, 145 (1983).

[3] I. Hofmann, Phys. Rev. E, 57(4), 4713 (1998).

[4] A.V. Fedotov, Proc. of the 2003 Part. Accel. Conf. (Portland, OR), p. 383.

[5] S. Bernal et al, Proc. of the 2005 Part. Accel. Conf. (Knoxville, TN), FOAD005.

[6] M. Reiser and H. Li, J. of Appl. Physics, 96, p. 784 (2004).

[7] R.A. Kishek, P.G. O'Shea, and M. Reiser, Phys. Rev. Lett, 21, 4514, (2000).

[8] S. Bernal et al, submitted to PRST-AB (2005). 